
JNM
J Neurogastroenterol Motil, Vol. 22 No. 4 October, 2016
pISSN: 2093-0879 elSSN: 2093-0887
http://dx.doi.org/10.5056/jnm15160

\title{
Prevalence of Irritable Bowel Syndrome-like Symptoms in Japanese Patients with Inactive Inflammatory Bowel Disease
}

\author{
Toshihiko Tomita, ${ }^{1}$ Yu Kato, ${ }^{1}$ Mayu Takimoto, ${ }^{1}$ Takahisa Yamasaki, ${ }^{1}$ Takashi Kondo, ${ }^{1}$ Tomoaki Kono, ${ }^{1}$ Katsuyuki Tozawa, \\ Yoko Yokoyama, ${ }^{2}$ Hisatomo Ikehara, ${ }^{1}$ Yoshio Ohda, ${ }^{1}$ Tadayuki Oshima, ${ }^{1}$ Hirokazu Fukui, ${ }^{1}$ Shigemi Tanaka, ${ }^{3}$ Masayuki Shima, ${ }^{4}$ \\ Jiro Watari, ${ }^{1}$ and Hiroto Miwa ${ }^{1 *}$ \\ Divisions of ${ }^{1}$ Gastroenterology and ${ }^{2}$ Inflammatory Bowel Disease, Department of Internal Medicine, Hyogo College of Medicine, Nishinomiya, \\ Hyogo, Japan; ${ }^{3}$ Tanaka Naika Clinic, Mikage, Hyogo, Japan; and ${ }^{4}$ Department of Public Health, Hyogo College of Medicine, Nishinomiya, \\ Hyogo, Japan
}

\section{Background/Aims}

Few studies are available that have investigated the risk factors for overlapping irritable bowel syndrome (IBS)-like symptoms in patients with inactive inflammatory bowel disease (IBD). The present study has 3 objectives: (1) to assess the prevalence of IBS-like symptoms in Japanese patients with inactive IBD using Rome III criteria, (2) to examine the relationship of IBS-like symptoms to health related quality of life (HR-QOL), and (3) to investigate associations for developing IBS-like symptoms in patients with inactive IBD.

\section{Methods}

IBS-like symptoms were evaluated using the Rome III questionnaire for functional gastrointestinal disorders. HR-QOL and hospital anxiety and depression scale were evaluated.

\section{Results}

IBS-like symptoms were found in 17.5\% (7/40) of patients with inactive ulcerative colitis, $27.1 \%$ (29/107) of patients with inactive Crohn's disease (CD), and 5.3\% (23/438) of healthy control subjects. The QOL level was significantly lower and anxiety score was significantly higher in inactive CD patients with IBS-like symptoms than in those without such symptoms $(P=0.003, P=0.009)$. Use of anti-anxiety drugs was associated with the presence of IBS symptoms $(P=0.045)$. HR-QOL score was lower and anxiety score was higher in patients with inactive ulcerative colitis, but the difference was not statistically significant.

\section{Conclusions}

The prevalence of IBS-like symptoms in inactive IBD patients was significantly higher than in healthy controls. Inactive CD patients with IBS-like symptoms has low QOL and anxiety; suggesting that anxiety may be associated with symptom development in such patients.

(J Neurogastroenterol Motil 2016;22:661-669)

Key Words

Anxiety; Inflammatory bowel diseases; Irritable bowel syndrome; Quality of life

Received: October 6, 2015 Revised: March 3, 2016 Accepted: March 20, 2016

(a) This is an Open Access article distributed under the terms of the Creative Commons Attribution Non-Commercial License (http://creativecommons. org/licenses/by-nc/4.0) which permits unrestricted non-commercial use, distribution, and reproduction in any medium, provided the original work is properly cited.

*Correspondence: Hiroto Miwa, MD, PhD

Division of Gastroenterology, Department of Internal Medicine, Hyogo College of Medicine, 1-1, Mukogawa-cho, Nishinomiya, Hyogo 663-8501, Japan

Tel: +81-798-45-6665, Fax: +81-798-45-6661, E-mail: miwahgi@hyo-med.ac.jp

Toshihiko Tomita and Yu Kato contributed equally to this work. 


\section{Introduction}

Inflammatory bowel disease (IBD), including ulcerative colitis (UC) and Crohn's disease (CD), is associated with intestinal tract abnormalities that may be immunological or organic in nature, and is accompanied by symptoms such as chronic diarrhea, abdominal pain, and bloody stool. The recent development and marketing of products such as anti-TNF- $\alpha$ agents and immunomodulatory drugs have resulted in dramatic progress in the medical treatment of inflammatory bowel disease. ${ }^{1-3}$ The objective of medical treatment has also evolved, from the induction of clinical remission to the healing of mucosal lesions. ${ }^{4}$ However, irritable bowel syndrome (IBS) can occur even in the absence of organic abnormalities, and can be associated with gastrointestinal symptoms such as diarrhea and constipation. ${ }^{5-7}$

Conventionally, IBS and IBD have been considered completely separate diseases. However, recent reports suggest that IBD can manifest IBS-like symptoms such as diarrhea and abdominal pain, even after bowel inflammation has been eliminated and the patient has entered clinical remission or mucosal lesions have healed. ${ }^{8-16}$ This has been attributed to the persistence of microinflammation in the mucosa and to increased permeability of the gastrointestinal mucosa. ${ }^{17}$ Such symptoms are thought to occur in approximately $30 \%$ to $50 \%$ of patients with inactive IBD, a considerably higher prevalence than in healthy control subjects. ${ }^{8-16}$ These findings are of considerable interest as we attempt to deepen our understanding of the inactive IBD disease state.

Currently, a number of reports are available on the prevalence of IBS complications, primarily focusing on European research. However, the reported studies suffer from a number of flaws, including no control group, small number of subjects, and lack of clarity in the definition of IBD remission and diagnostic procedures. In addition, most of these studies used the Rome II definition; only a few Rome III reports are available. ${ }^{10,12,13}$

In Japan, the prevalence of IBS complications in IBD has not been investigated on a large scale, so little is known about whether such complications are actually common among Japanese patients, and risk factors that may predispose patients to IBS-like symptoms have not been addressed.

We thus decided to investigate 3 objectives: (1) to assess the prevalence of IBS-like symptoms in Japanese patients with inactive IBD using the Rome III criteria, (2) to examine the relationship of IBS-like symptoms to health related quality of life (HR-QOL), and (3) to investigate the associations for developing IBS-like symptoms in patients with inactive IBD.

\section{Materials and Methods}

\section{Patients}

Inactive IBD patients were consecutively enrolled in this study from April 2012 to December 2014. The remission stage of IBD was defined as follows. CD: Crohn's disease activity index (CDAI) index $\leq 150$; UC: clinical activity index (CAI) for the evaluation of patients with $\mathrm{UC} \leq 4$ and $\mathrm{C}$-reactive protein $(\mathrm{CRP}) \leq 0.3{ }^{17,18}$ $\mathrm{CDAI}$ and CAI are clinical disease activity indices which is determined by the clinical symptoms and several parameters including bowel habits, general condition, body weight, hemoglobin level and so on, but not endoscopic findings. In this present study, the patients' clinical information and symptoms were evaluated using a self-completed symptom questionnaire.

The healthy control group consisted of 500 employees, randomly selected from among staff members of 5 local companies in Hyogo Prefecture. These patients and members of the health control group were surveyed by self-administered questionnaire.

This study was approved by the Ethics Committee of the Hyogo College of Medicine. Written informed consent was obtained from all patients prior to their enrollment in the study. We also obtained written consent from the staff members of local businesses who made up our control group.

\section{Questionnaires}

IBS-like symptoms were evaluated using a Japanese version of the Rome III diagnostic questionnaire for functional gastrointestinal disorders. ${ }^{5}$ HR-QOL scores were evaluated for general HR-QOL (eg, 8-item short-form health survey [SF-8]: physical and mental component summary) and disease-specific QOL (Inflammatory bowel disease questionnaire: IBDQ). ${ }^{19,20}$ Tendencies toward depression and anxiety were assessed on the hospital anxiety and depression scale (HADS); values were calculated for depression and anxiety, and for the total overall score. ${ }^{21,22}$ We then compared the control group and the inactive IBD patients based on the prevalence of IBS-like symptoms, findings for QOL, and scores for depression and anxiety. We also conducted a retrospective review of medical records, and compared age, gender, body mass index, operation history, duration of disease, location of disease, history of smoking, treatment history, and clinical disease activity index (CAI and CDAI) between the 2 groups (those with and without IBS-like symptoms). 


\section{Statistical Methods}

All results are expressed as mean \pm standard deviation. The Mann-Whitney U test, the Chi-square test, and Fisher's exact test were used to compare the 2 groups. Statistical significance was defined as a value of $P<0.05$. Statistical analysis was performed using SPSS for Windows (Version 12.0; SPSS, Inc, Chicago, IL, USA).

\section{Results}

Of the 147 enrolled patients with inactive IBD, 107 had inactive CD and 40 had inactive UC. The 500 people who served as control subjects were randomly selected from among the employees of 5 local companies. All patients and subjects completed a selfadministered questionnaire.

\section{Characteristics of the Inactive Inflammatory Bowel Disease Patients (see Table 1)}

Of the 500 control subjects, 62 failed to complete the questionnaire as instructed, and were excluded from consideration. Data was analyzed from a total of 438 control subjects and 147 patients (CD: 107, UC: 40). Mean CDAI was 95.6 in the inactive CD group, and mean CAI was 1.2 in the inactive UC group. Mean age was $38.5 \pm 11.1$ years in the control group, $38.7 \pm 10.2$ years in the CD group and $41.1 \pm 11.4$ years in the UC group. There was no statistical significance. Men accounted for $60.7 \%$ (238/438 subjects) in the control group, $75.5 \%$ (80/107 patients) in the CD group, and $72.5 \%$ (29/40 patients) in the UC group. BMI was $22.3 \pm 3.3$ in the control group, $20.9 \pm 3.3$ in the CD group and $20.8 \pm 1.9$ in the UC group. There was a history of smoking in $4.6 \%$ of the control group, $8.4 \%$ of the CD group and $20.0 \%$ of the UC group. The duration of disease was $10.0 \pm 7.6$ years in the CD group and $8.6 \pm 7.2$ years in the UC group. These results did not reach significant difference.

\section{Characteristics of Crohn's Disease and Ulcerative colitis Patients with and Without Irritable Bowel Syndrome-like Symptoms}

Duration of disease was significantly longer in $\mathrm{CD}$ patients with IBS-like symptoms $(16.0 \pm 8.5$ years $)$ than in those without IBS-like symptoms $(11.0 \pm 6.7$ years $)(P=0.021)$. Use of antianxiety drugs was associated with the presence of IBS symptoms ( $P$ $=0.045$ ) (Table 2). In UC patients, use of anti-anxiety drugs were also correlated with IBS-like symptoms (Table 3).

\section{Prevalence of Irritable Bowel Syndrome-like Symptoms According to the Rome III Criteria (see Fig. 1)}

Using the Rome III criteria, IBS-like symptoms were found in $27.1 \%(29 / 107)$ of patients with inactive CD and in $17.5 \%(7 / 40)$ of patients with inactive UC, but in only 5.3\% (23/438) of healthy control subjects. All of the subtypes of IBS like symptoms were IBS-diarrhea predominant.

Table 1. Characteristics of the Inactive Inflammatory Bowel Disease Patients

\begin{tabular}{lccc}
\hline & Controls & Inactive CD & Inactive UC \\
\hline Number & 438 & 107 & 40 \\
Age (yr) & $38.5 \pm 11.1$ & $38.7 \pm 10.2$ & $41.4 \pm 11.4$ \\
Gender, male (n) & $238(60.7 \%)$ & $80(75.5 \%)$ & $29(72.5 \%)$ \\
BMI & $22.3 \pm 3.3$ & $20.9 \pm 3.3$ & $20.8 \pm 1.9$ \\
Operation history (n) & $0(0.0 \%)$ & Ileal resection: 14 cases & $0(0.0 \%)$ \\
& & Ileocecal resection: 19 cases & $8.6 \pm 7.2$ \\
Duration of disease (yr) & - & Colonic resection: 11 cases & $8(20.0 \%)$ \\
Smoking (n) & $20(4.6 \%)$ & $10.0 \pm 7.6$ & $9(8.4 \%)$ \\
CRP & - & $0.12 \pm 0.2$ & $0.06 \pm 0.1$ \\
CDAI & - & $95.6 \pm 53.5$ & - \\
CAI & - & - & $1.2 \pm 1.3$
\end{tabular}

$\mathrm{CD}$, Crohn's disease; UC, ulcerative colitis; BMI, body mass index; CRP, C-reactive protein; CDAI, CD activity index; CAI, clinical activity index. Data are expressed as mean $\pm \mathrm{SD}$ 
Table 2. Characteristics of Crohn's Disease Patients with and Without Irritable Bowel Syndrome-like Symptoms

\begin{tabular}{|c|c|c|c|}
\hline Inactive CD & $\begin{array}{l}\operatorname{IBS}(+) \\
(\mathrm{n}=29)\end{array}$ & $\begin{array}{c}\text { IBS }(-) \\
(\mathrm{n}=78)\end{array}$ & $P$-value \\
\hline Age & $39.7 \pm 9.1$ & $38.3 \pm 10.7$ & 0.379 \\
\hline Sex (male/female) & $22 / 7$ & $60 / 18$ & 1.000 \\
\hline BMI & $21.5 \pm 4.3$ & $22.3 \pm 12.1$ & 0.769 \\
\hline Duration of disease (yr) & $16.0 \pm 8.5$ & $11.0 \pm 6.7$ & 0.021 \\
\hline Disease location (proctitis/left/total colitis) & $6 / 17 / 6$ & $15 / 50 / 13$ & 0.854 \\
\hline \multirow[t]{4}{*}{ Operation history } & $12(41.4 \%)$ & $32(41.0 \%)$ & 0.384 \\
\hline & Ileal resection: 3 cases & Ileal resection: 6 cases & \\
\hline & Ileocecal resection: 8 cases & Ileocecal resection: 22 cases & \\
\hline & Colonic resection: 1 case & Colonic resection: 4 cases & \\
\hline \multicolumn{4}{|l|}{ Disease treatment } \\
\hline 5-ASA/sulfasalazine & 10 & 27 & 1.000 \\
\hline Steroid & 0 & 0 & - \\
\hline $\mathrm{AZA} / 6 \mathrm{MP}$ & 5 & 22 & 0.320 \\
\hline Anti-TNF & 20 & 43 & 0.270 \\
\hline Anti-anxiety drug & $4(13.8 \%)$ & $2(2.5 \%)$ & 0.045 \\
\hline Smokers & $5(17.2 \%)$ & $8(10.3 \%)$ & 0.515 \\
\hline CDAI & $115.4 \pm 49.6$ & $97.2 \pm 58.2$ & 0.141 \\
\hline
\end{tabular}

$\mathrm{CD}$, Crohn's disease; IBS, irritable bowel syndrome; BMI, body mass index; 5-ASA, 5-aminosalicylate; AZA, azathioprine; 6-MP, 6-mercaptopurine; TNF, tumor necrosis factor; CDAI, CD activity index.

Data are expressed as mean $\pm \mathrm{SD}$.

Table 3. Characteristics of Ulcerative Colitis Patients with and Without Irritable Bowel Syndrome-like Symptoms

\begin{tabular}{lccc}
\hline \multicolumn{1}{c}{ Inactive UC } & $\begin{array}{c}\text { IBS }(+) \\
(\mathrm{n}=7)\end{array}$ & $\begin{array}{c}\text { IBS }(-) \\
(\mathrm{n}=33)\end{array}$ & $P$-value \\
\hline Age & $46.2 \pm 15.7$ & $40.6 \pm 10.2$ & 0.393 \\
Sex (male/female) & $2 / 5$ & $19 / 14$ & 0.225 \\
BMI & $20.7 \pm 1.6$ & $20.9 \pm 1.9$ & 0.984 \\
Duration of disease (yr) & $12.1 \pm 9.6$ & $7.9 \pm 6.6$ & 0.275 \\
Disease location (proctitis/left/total colitis) & $3 / 3 / 1$ & $9 / 16 / 8$ & 0.540 \\
Operation history & $0(0 \%)$ & $0 \%)$ & - \\
Disease treatment & & 32 & - \\
5-ASA/sulfasalazine & 7 & 0 & - \\
Steroid & 0 & 0 & 1.000 \\
AZA/6MP & 0 & $9 / 16 / 8$ & \\
Anti-TNF & 0 & $1(0.03 \%)$ & 0.540 \\
Disease location (proctitis/left/total colitis) & $3 / 3 / 1$ & $9(27.2 \%)$ & 0.012 \\
$\quad$ Anti-anxiety drug & $3(42.8 \%)$ & $1.8 \pm 1.8$ & 1.000 \\
Smokers & $2(33.3 \%)$ & 0.956 & \\
CAI & $1.8 \pm 2.0$ & & \\
\hline
\end{tabular}

UC, ulcerative colitis; IBS, irritable bowel syndrome; BMI, body mass index; 5-ASA, 5-aminosalicylate; AZA, azathioprine; 6-MP, 6-mercaptopurine; TNF, tumor necrosis factor; CAI, clinical activity index.

Disease-specific Quality of Life Score in Inactive Inflammatory Bowel Disease (Crohn's Disease and Ulcerative Colitis) Patients with and Without IBS-like Symptoms (see Fig. 2)

Disease-specific QOL (IBDQ) score level was significantly lower in CD and UC patients with IBS-like symptoms than in those without such symptoms $(165.0 \pm 21.0$ vs $183.6 \pm 29.0 ; P=$ $0.003,177.5 \pm 16.1$ vs $190.0 \pm 14.2 ; P=0.031)$. 
Health Related Quality of Life (SF-8) Score in Inactive Crohn's Disease Patients with and Without Irritable Bowel Syndrome-like Symptoms (see Fig. 3)

General QOL (physical and mental summary scores) was significantly lower in CD patients with IBS-like symptoms than in those without such symptoms $(49.8 \pm 5.2$ vs $52.0 \pm 3.9 ; P=0.031$, $47.1 \pm 6.2$ vs $49.5 \pm 6.3, P=0.028)$. General QOL score was

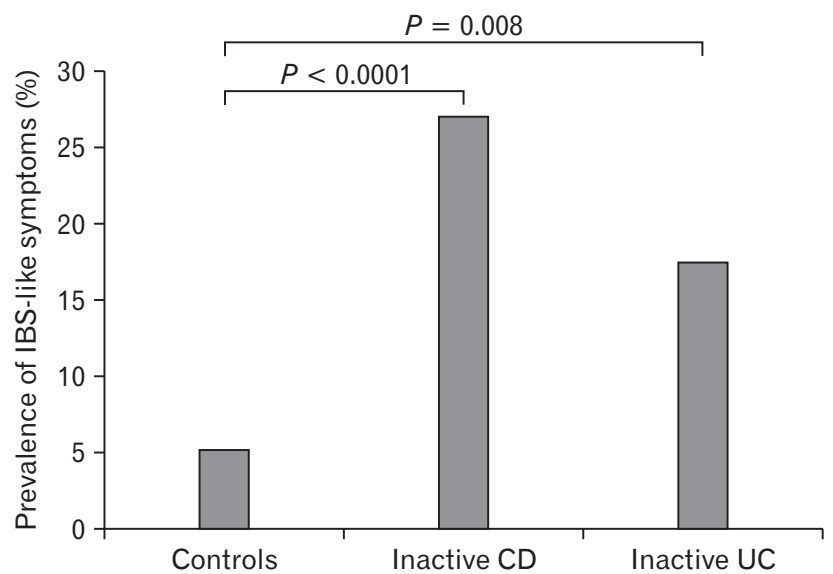

Figure 1. Prevalence of irritable bowel syndrome (IBS)-like symptoms according to the Rome III criteria. IBS-like symptoms were found in $27.1 \%$ (29/107) of patients with inactive Crohn's disease (CD) and in $17.5 \%(7 / 40)$ of patients with inactive ulcerative colitis (UC), but in only 5.3\% (23/438) of healthy control subjects.

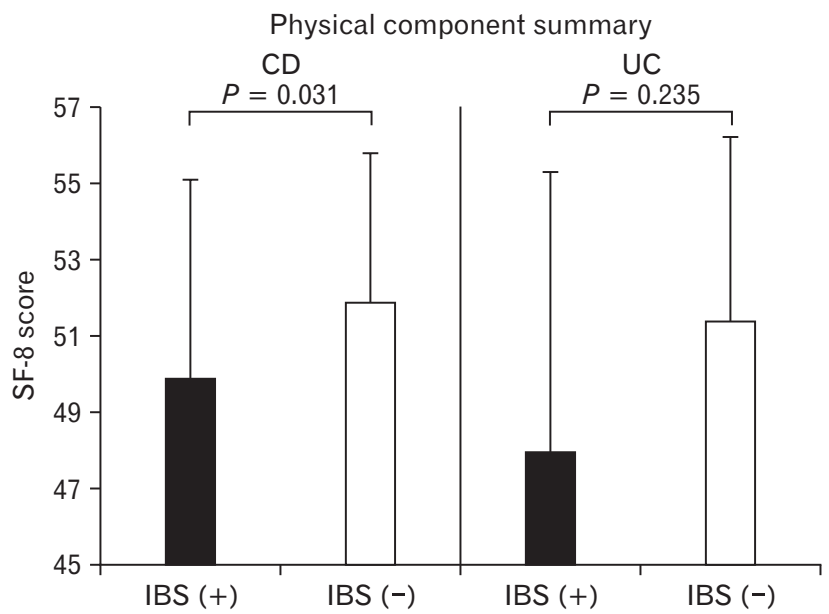

also lower in UC patients with IBS-like symptoms than in those without such symptoms, however no differences were found in inactive UC patients with or without IBS-like symptoms.

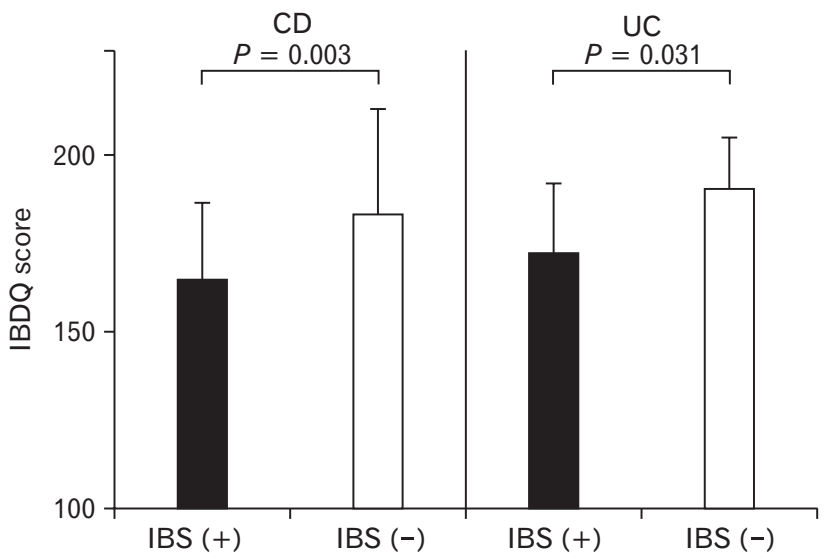

Figure 2. Disease-specific quality of life (QOL) (eg, inflammatory bowel disease questionnaire [IBDQ]) score in inactive IBD patients with and without irritable bowel syndrome (IBS)-like symptoms. Disease-specific QOL (IBDQ) score was significantly lower in Crohn's disease (CD) and ulcerative colitis (UC) patients with IBS-like symptoms than in those without such symptoms. Statistical analyses were assessed by the Mann-Whitney U test.

Figure 3. General quality of life (QOL) (eg, 8-item short-form health survey [SF-8]) score in inactive inflammatory bowel disease (IBD) patients with and without irritable bowel syndrome (IBS) like symptoms. General QOL (physical and mental summary scores) was significantly lower in Crohn's disease (CD) patients with IBS-like symptoms than in those without such symptoms. In inactive ulcerative colitis (UC) patients, the general QOL score was lower in UC patients with IBS-like symptoms than in those without such symptoms, but that difference was not statistically significant. Statistical analyses were assessed by the Mann-Whitney U test. 

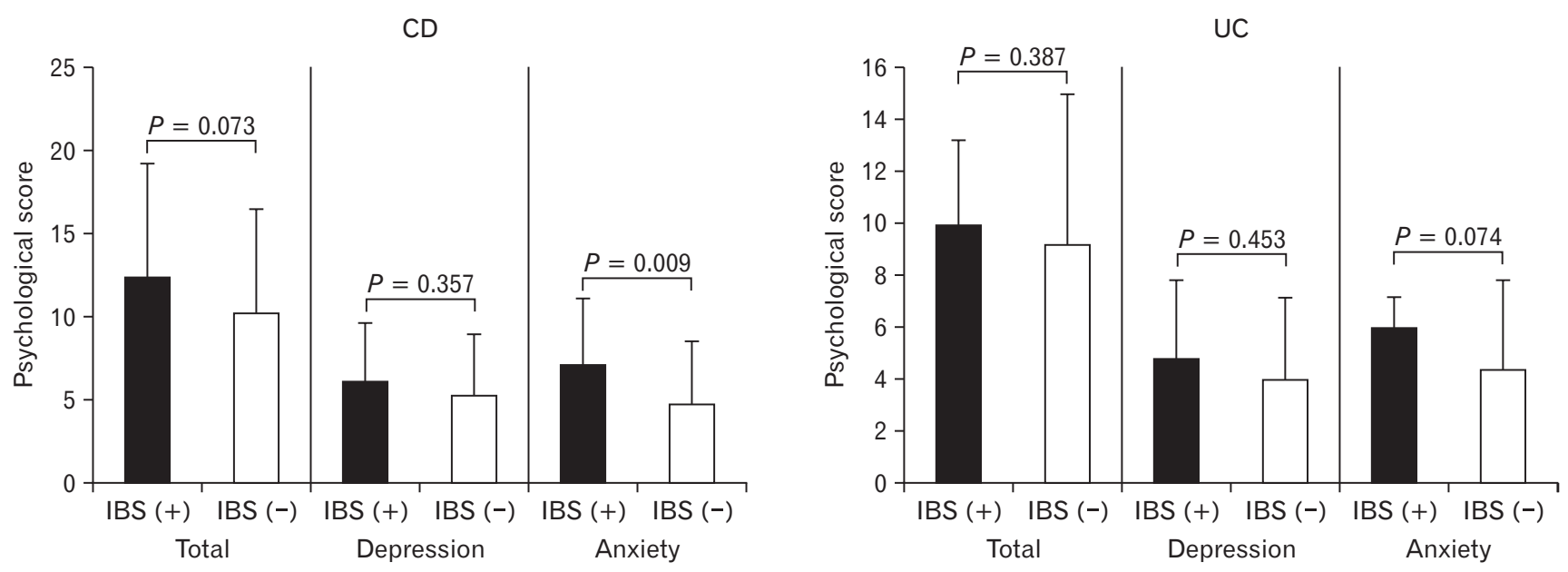

Figure 4. Psychological score (eg, hospital anxiety and depression scale) in inactive inflammatory bowel disease patients with and without irritable bowel syndrome (IBS)-like symptoms. Among inactive Crohn's disease (CD) and ulcerative colitis (UC) patients, total psychological score was higher in those with IBS-like symptoms than in those without IBS like symptoms, but that difference was not statistically significant. The anxiety score was significantly higher in inactive CD patients with IBS-like symptoms than in those without such symptoms.

\section{Psychological Scores (HADS) in Inactive Inflammatory Bowel Disease Patients with and Without Irritable Bowel Syndrome-like Symptoms (see Fig. 4)}

Among inactive CD patients, total psychological score (HADS) was higher in those with IBS-like symptoms than in those without IBS like symptoms $(12.4 \pm 6.8$ vs $10.2 \pm 6.3, P=0.073)$. No such differences were found in the inactive UC patients $(10.0 \pm$ 3.2 vs $9.2 \pm 5.7, P=0.387)$. The anxiety score was significantly higher in inactive CD patients with IBS-like symptoms than in those without such symptoms $(7.0 \pm 4.0$ vs $4.9 \pm 3.6, P=0.009)$. However, there was no significant difference in depression score between inactive $\mathrm{CD}$ patients with or without IBS-like symptoms (6.1 \pm 3.6 vs $5.4 \pm 3.5, P=0.357$ ).

Generally, the cut-off level of 8/21 in HADS was used to examine the patients who have anxiety and depression. ${ }^{22}$ In the present study of anxiety score, over value of cut-off was more seen in $\mathrm{CD}$ patients with IBS-like symptoms than without such symptoms $(82.7 \%$ vs $55.8 \%, P=0.027)$.

The psychological factor score was also higher in patients with inactive UC who had IBS-like symptoms than in those patients without such symptoms, but the difference was not statistically significant $(6.0 \pm 1.1$ vs $4.4 \pm 3.4, P=0.074)$.

\section{Discussion}

This paper reports the results of our investigation of complications of IBS-like symptoms in Japanese patients with IBD. We used the Rome III criteria to investigate both the prevalence of complications with IBS-like symptoms and the risk factors for such complications. Up to this point, the prevalence of IBS-like symptoms as complications of inactive IBD has been reported primarily in European and American populations. Those reports have included the following findings: $35.4 \%$ (11/31), 45.6\% (42/92), 59.7\% (37/62), and $57 \%$ (23/40) of CD patients; $38 \%$ (7/18), $46 \%$ (23/50), 38.6\% (17/44), and $33 \%(14 / 43)$ of UC patients. ${ }^{10-13}$ In healthy adults, the prevalence of IBS using the Rome III diagnostic questionnaire was reported to be approximately $10 \%$ to $15 \% .^{23-25}$ The Rome III classification is based on the symptom-based diagnostic criteria which is not explained by pathologically and endoscopically based disorders. Regarding the Rome III classification, the duration for the diagnosic criteria originates at 6 months prior to clinical presentation, and diagnosis and must be currently active for 3 months. This duration is less defenitive than Rome II (12 weeks of symptoms over 12 months) and is easier to understand in a clinical setting questionnaire.

Higher values could certainly be expected for the prevalence of IBS complications in patients with inactive IBD. However, the reported studies had no control groups and were not consistent in the definition of inactive IBD or in the methods used for the diagnosis of IBS. ${ }^{10-13}$ As a result, the true prevalence of IBS-like symptoms remained uncertain.

We believe that our methodology has several advantages over that used in previous reports. (1) Inactive IBD was strictly defined. (2) Clear criteria were established for the control group. (3) Factors 
such as the mechanism of onset of IBS symptoms were considered.

Our research in patients with inactive IBD showed a $27.1 \%$ prevalence of complications with IBS-like symptoms for CD and $17.5 \%$ for UC, which was lower than reported in Europe and America. One possible reason for this difference is the lack of uniformity in the definition of inactive IBD in the previous studies. For example, in the report from Ansari et $\mathrm{al}^{12}$ in Iran, inactive UC was defined by number of bowel movements, the presence or absence of bloody stool, mucosal findings, and overall physician assessment based on the Mayo index, with no assessment of parameters such as serum CRP. Keohane et al $^{9}$ defined inactive IBD as a CRP value of 1.0 or below. However, the incidence of IBS complications can be greatly influenced by the presence of residual microinflammation in the mucosa, so the possibility of a higher value cannot be ruled out.

Another possible reason could be differences in IBS diagnostic procedures. Simren et al reported the use of the gastrointestinal symptom rating scale to assess IBS-like symptoms, while Keohane et $\mathrm{al}^{9}$ and Minderhoud et $\mathrm{al}^{14}$ used the Rome II criteria. ${ }^{9,11,14}$ Here, we have defined inactive IBD as CDAI $\leq 150$ for $\mathrm{CD}$, and as CAI $\leq 4$ points for $\mathrm{UC}$ and $\mathrm{CRP} \leq 0.3$ for both $\mathrm{UC}$ and $\mathrm{CD}$, and we based our diagnosis of IBS on the Rome III criteria. The prevalence of IBS symptoms was $5.3 \%$ in our control group, in good agreement with previous reports. We found IBS-like symptoms in $27.1 \%$ of patients with inactive CD and in $17.5 \%$ of patients with inactive UC. These findings suggest that IBS symptoms are 3 to 6 times more likely to occur in patients with inactive IBD than in the ordinary population.

Additionally, very little work has been done yet in Japan on these topics, so the prevalence of IBS in inactive IBD is not well understood. One recent report is available on the prevalence of IBS-like symptoms as complications during inactive UC. They reported a higher prevalence of complications (26.7\%) than that of our study. ${ }^{8}$ However, that report used an endoscopic index and defined "inactive" as $\leq$ Matts grade 2. If inactive UC is defined as $\leq$ Matts grade 1, the prevalence of IBS-like symptoms in inactive UC patients in that report was $15.4 \%$, similar to the findings from our research. We feel that the definition of "inactive" in that report is problematic, since obvious inflammation remains at Matt grade 2. The definition of "inactive" used in our investigation is based on CAI and CRP findings, and reflects a disease state in which inflammatory cell infiltration is almost entirely absent, even at the histological level. We believe that this is an appropriate definition for such research, and that our results represent the actual incidence of IBS symptoms.

Almost all previous studies have reported a higher rate of IBS- like complications with $\mathrm{CD}$ than with UC. In our research, we were able to apply the same criteria to both $\mathrm{CD}$ and $\mathrm{UC}$ in a relatively large population of subjects, and our results showed that these complications developed approximately twice as often in $\mathrm{CD}$ patients than in patients with UC. The reasons for the higher rate in $\mathrm{CD}$ than in UC patients remain poorly understood. However, factors such as the area affected by lesions, including the small intestine, may be involved.

In this research, we used the IBDQ for disease activity HRQOL and the SF-8 for ordinary HR-QOL, and compared findings for IBD patients with and without IBS symptoms. The IBDQ score was significantly lower for both CD and UC patients with IBS symptoms than for those without such symptoms. Both physical and psychological findings from the SF-8 for ordinary QOL were significantly lower in CD patients with IBS-like symptoms than in those without such symptoms. Because psychological factors are known to strongly influence health-related QOL, we used the HADS to assess depression and anxiety. ${ }^{26}$ Particularly in patients with $\mathrm{CD}$, the total HADS tended to be higher with IBSlike symptoms than without such symptoms, and within that group IBS-like symptoms were particularly common among the patients who showed strong anxiety traits. Depressive episodes and anxiety states are known comorbidities of IBS. The occurrence of IBS-like symptoms is thus particularly significant in IBD patients with clinically inactive disease who are prone to depression and anxiety. Our investigation clearly showed a strong tendency toward not depression but anxiety in patients with inactive IBD and IBS-like symptoms, especcially in CD patients. However, it is unclear whether IBS symptoms are likely to develop in patients who have a strong underlying tendency toward anxiety, or whether the occurrence of IBS strengthens the tendency toward anxiety. In order to determine whether psychological comorbidity was preexistant in patients with inactive IBD who developed IBS, we investigated each patient's history of antianxiety drug use before IBD became inactive. Our results showed a significantly higher rate of occurrence of IBSlike symptoms in those patients with inactive CD who had used antianxiety drugs before their disease became inactive. At present the mechanism of onset of IBS-like symptoms as a complication of IBD remains poorly understood. However, we are quite interested in this new finding that patients with a strong tendency toward anxiety are more likely to develop IBS symptoms.

Generally, the occurrence of IBS symptoms in a patient with inactive IBD is considered proof of a relationship between residual microinflammation and symptom onset. ${ }^{26-28}$ Functional gastrointestinal disorders have been reported to occur following Salmonella-in- 
duced infectious enteritis, indicating a correlation between symptom onset and the presence of residual microinflammation. ${ }^{29,30}$ Keohane et $\mathrm{al}^{9}$ have reported that levels of the microinflammation marker fecal calprotectin in IBD patients are significantly higher in the presence of IBS symptoms than in the absence of such symptoms. ${ }^{31-33}$ This reports provides further background for the relaionship between IBS and inflammation. In this present research, our invesigation of background factors showed that the duration of disease was significantly longer in CD patients with IBS-like symptoms. This long-term residual microinflammation may also be related to the onset of IBS symptoms. Further long-term follow up study will be needed to clarify the associatetion between residual microinflammation and IBS symptom generation.

The present study has some potential limitations. First, this was a retrospective study from a single institution. It will be important to conduct a prospective study to exclude some bias of patient's background factors. Second, we did not evaluate the microinflammation marker in this study. Regarding the pathophysiology of IBS symptoms in a patient with inactive IBD, previous studies reported that residual microinflammation may affect the occurrence of IBS symptoms. It is meaningfull to check the mucosal change by biopsy in the study of the pathophisiology of IBS symptoms. Third, the sample size in UC was ralatively not enough, which may affect the results.

In conclusion, we clarified that (1) the prevalence of IBS-like symptoms in inactive Japanese IBD patients was significantly higher than in healthy controls and (2) inactive CD patients with IBSlike symptoms has low QOL and anxiety, suggesting that anxiety may be associated with symptom development in such patients.

\section{Financial support: None.}

\section{Conflicts of interest: None.}

Author contributions: Toshihiko Tomita and Yu Kato contributed to the concept and design of the study; Toshihiko Tomita, Mayu Takimoto, Takahisa Yamasaki, Takashi Kondo, Tomoaki Kono, Katsuyuki Tozawa, Yoko Yokoyama, Hisatomo Ikehara, Yoshio Ohda, Hirokazu Fukui, and Shigemi Tanaka were involved in data acquisition and analysis; Tadayuki Oshima and Hirokazu Fukui provided statistifical design; Jiro Watari and Hiroto Miwa coordinated acquisition of data and trial supervision; and all authors contributed to data interpretation, critically reviewed the manuscript for important intellectual contents, and approved final version of the manuscript.

\section{References}

1. Kucharzik T, Maaser C, Lügering A, et al. Recent understanding of IBD pathogenesis: implications for future therapies. Inflamm Bowel Dis 2006;12:1068-1083.

2. Rutgeerts P, Sandborn WJ, Feagan BG, et al. Infliximab for induction and maintenance therapy for ulcerative colitis. $\mathrm{N}$ Engl J Med 2005;353:2462-2476.

3. Reinisch W, Sandborn WJ, Hommes DW, et al. Adalimumab for induction of clinical remission in moderately to severely active ulcerative colitis: results of a randomised controlled trial. Gut 2011;60:780-787.

4. Khanna R, Bouguen G, Feagan BG, et al. A systematic review of measurement of endoscopic disease activity and mucosal healing in Crohn's disease: recommendations for clinical trial design. Inflamm Bowel Dis 2014;20:1850-1861.

5. Longstreth GF, Thompson WG, Chey WD, Houghton LA, Mearin F, Spiller RC. Functional bowel disorders. Gasroenterology 2006;130:14801491

6. Spiller R, Aziz Q, Creed F, et al. Guidelines on the irritable bowel syndrome: mechanisms and practical management. Gut 2007;56:17701798.

7. Khan S, Chang L. Diagnosis and management of IBS. Nat Rev Gastroenterol Hepatol 2010;7:565-581

8. Fukuba N, Ishihara S, Tada Y, et al. Prevalence of irritable bowel syndrome-like symptoms in ulcerative colitis patients with clinical and endoscopic evidence of remission: prospective multicenter study. Scand J Gastroenterol 2014;49:674-680.

9. Keohane J, O’Mahony C, O’Mahony L, O’Mahony S, Quigley EM, Shanahan F. Irritable bowel syndrome-type reflection of occult inflammation? Am J Gastroenterol 2010;105:1788-1794.

10. Piche T, Ducrotté P, Sabate JM, et al. Impact of functional bowel symptoms on quality of life and fatigue in quiescent Crohn disease and irritable bowel syndrome. Neurogastroenterol Motil 2010;22:626-e174.

11. Simrén M, Axelsson J, Gillberg R, O’Mahony S, Quigley EM, Shanahan F. Quality of life in inflammatory bowel disease in remission: the impact of IBS-like symptoms and associated psychological factors. Am J Gastroenterol 2002;97:389-396.

12. Ansari R, Attari F, Razjouyan H, et al. Ulcerative colitis and irritable bowel syndrome: relationships with quality of life. Eur J Gastroenterol Hepatol 2008;20:46-50.

13. Vivinus-Nébot M, Frin-Mathy G, Bzioueche H, et al. Functional bowel symptoms in quiescent inflammatory bowel diseases: role of epithelial barrier disruption and low-grade inflammation. Gut 2014;63:744-752.

14. Minderhoud IM, Oldenburg B, Wismeijer JA, et al. IBS-like symptoms in patients with inflammatory bowel disease in remission; relationships with quality of life and coping behavior. Dig Dis Sci 2004;49:469-474.

15. Halpin SJ, Ford AC. Prevalence of symptoms meeting criteria for irritable bowel syndrome in inflammatory bowel disease: systematic review and meta-analysis. Am J Gastroenterol 2012;107:1474-1482.

16. Farrokhyar F, Marshall JK, Easterbrook B, Irvine EJ. Functional gastrointestinal disorders and mood disorders in patients with inactive inflammatory bowel disease: prevalence and impact on health. Inflamm Bowel 
Dis 2006;12:38-46.

17. Vivinus-Nébot M, Frin-Mathy G, Bzioueche H, et al. Functional bowel symptoms in quiescent inflammatory bowel diseases: role of epithelial barrier disruption and low-grade inflammation. Gut 2014;63:744-752.

18. Walmsley RS, Ayres RC, Pounder RE, Allan RN. A simple clinical colitis activity index. Gut 1998;43:29-32.

19. Russel MG, Pastoor CJ, Brandon S, et al. Validation of the Dutch translation of the Inflammatory Bowel Disease Questionnaire (IBDQ): a health-related quality of life questionnaire in inflammatory bowel disease. Digestion 1997;58:282-288.

20. Guyatt G, Mitchell A, Irvine EJ, et al. A new measure of health status for clinical trials in inflammatory bowel disease. Gastroenterology 1989;96:804-810.

21. Zigmond AS, Snaith RP. The hospital anxiety and depression scale. Acta Psychiatr Scand 1983;67:361-370.

22. Snaith RP. The hospital anxiety and depression scale. Health Qual Life Outcomes 2003;1:29.

23. Miwa H. Prevalence of irritable bowel syndrome in Japan: Internet survey using Rome III criteria. Patient Prefer Adherence 2008;2:143-147.

24. Kaji M, Fujiwara Y, Shiba M, et al. Prevalence of overlaps between GERD, FD and IBS and impact on health-related quality of life. J Gastroenterol Hepatol 2010;25:1151-1156.

25. Jung HK, Halder S, McNally M, et al. Overlap of gastro-oesophageal reflux disease and irritable bowel syndrome: prevalence and risk factors in the general population. Aliment Pharmacol Ther 2007;26:453-461.

26. Jonefjäll B, Strid H, Ohman L, Svedlund J, Bergstedt A, Simren M. Characterization of IBS-like symptoms in patients with ulcerative colitis in clinical remission. Neurogastroenterol Motil 2013;25:756-e578.

27. Shanahan F. Enteric neuropathophysiology and inflammatory bowel disease. Neurogastroenterol Motil 1998;10:185-187.

28. Mawe GM, Collins SM, Shea-Donohue T. Changes in enteric neural circuitry and smooth muscle in the inflamed and infected gut. Neurogastroenterol Motil 2004;16(suppl 1):133-136.

29. Fock KM. Functional dyspepsia, H. pylori and post infectious FD. J Gastroenterol Hepatol 2011;26(suppl 3):39-41.

30. Cremon C, Stanghellini V, Pallotti F, et al. Salmonella gastroenteritis during childhood is a risk factor for irritable bowel syndrome in adulthood. Gastroenterology 2014;147:69-77.

31. Schoepfer AM, Beglinger C, Straumann A, et al. Ulcerative colitis: correlation of the Rachmilewitz endoscopic activity index with fecal calprotectin, clinical activity, C-reactive protein, and blood leukocytes. Inflamm Bowel Dis 2009;15:1851-1858.

32. Konikoff MR, Denson LA. Role of fecal calprotectin as a biomarker of intestinal inflammation in inflammatory bowel disease. Inflamm Bowel Dis 2006; 12:524-534.

33. Røseth AG, Fagerhol MK, Aadland E, Schjønsby H. Assessment of the neutrophil dominating protein calprotectin in feces. A methodologic study. Scand J Gastroenterol 1992;27:793-798. 